\title{
Response of the fetal heart to changes in load: from hyperplasia to heart failure \\ H M Gardiner
}

$\mathrm{T}$ he fetal circulation works in parallel with the dominant right ventricle, ejecting approximately $60 \%$ of the combined ventricular output. Three important communications exist between the two circulations (oval foramen and the arterial and venous ducts) that influence loading conditions. In particular, the determinants of fetal left ventricular filling differ considerably from those seen in the adult heart. Pulmonary venous return contributes only a small proportion to left ventricular preload because of the relatively low pulmonary blood flow in fetal life (rising from an estimated $11 \%$ to $25 \%$ of right ventricular output by the third trimester). ${ }^{1}$ Left ventricular filling depends predominantly on patency of the oval foramen to allow the relatively oxygen-rich blood returning from the placental circulation, via the umbilical vein and venous duct, to stream through the right atrium and enter the left side of the heart. ${ }^{2}$ The right ventricle fills from mostly upper body systemic venous return and the majority of its output is diverted away from the pulmonary circulation, via the arterial duct, to the descending aorta and thence to the placenta via the umbilical arteries. Hence the fetal right ventricle pumps against the systemic pressures of the lower fetal body and placental impedance, while the left ventricle ejects against the relatively high impedance of the fetal brain and upper body. Not surprisingly, ventricular loading is affected by the maturation of structures such as the placenta and changes in the impedance of the cerebral and placental vascular beds in response to hypoxaemia, as well as circulatory adaptations made by the fetus in response to intracardiac malformations.

\section{MATURATIONAL CHANGES IN LOADING}

Loading patterns alter throughout gestation; the ventricular pressure-volume loops of the chick embryo show an active response to increasing preload as the early myocardium organises. ${ }^{3}$ Mid trimester studies in the human fetus demonstrate the importance of trophoblastic invasion in the remodelling of the placental spiral arteries to create a low impedance placental circulation against which the right ventricle ejects. ${ }^{4}$ This low resistance circuit is pivotal to permit appropriate fetal growth as the right ventricle (unlike the left) responds to increasing afterload with a fall in ventricular output. Left ventricular preload becomes more dependent on pulmonary venous return in the third trimester as the pulmonary circulation increases and the oval foramen becomes relatively restrictive, thus reducing the right to left shunt. This leads to volume loading of right sided structures that is usually well tolerated in later pregnancy and regresses after delivery with no apparent long term effects. Preparation for postnatal closure of the arterial duct is effected by "cushions" of tissue seen at the pulmonary end of the duct towards term. These contribute to the increasing afterload faced by the fetal right ventricle as a consequence of placental changes near term and may lead to increased systemic venous pressure in the fetus.
The most dramatic changes in loading occur at birth when there is a sudden increase in distal impedance associated with removal of the placental circulation, a sixfold increase in pulmonary blood flow leading to a rise in left atrial pressure and reversal or cessation of flow through the oval foramen, and a more gradual closure of the arterial duct. This new serial arrangement of the circulation demands that both ventricles are suddenly able to receive and eject the entire cardiac output that was previously shared between them.

\section{PREMATURE CLOSURE OF FETAL SHUNTS}

Premature closure of fetal shunts can have serious consequences and suggests that myocardial maturation is critical in preparing the fetal heart to cope with the alterations of pressure and volume loading associated with delivery and successful adaptation to extrauterine life.

The most serious consequence of premature closure of the oval foramen in the first trimester fetus may be the development of hypoplastic left heart syndrome (aortic and mitral atresia) thought to occur because of reduced left ventricular preload. Closure during the second trimester is associated with right ventricular volume and pressure loading, increased systemic venous pressures, and fetal hydrops (characterised by increased fluid accumulation in the abdominal and thoracic cavities and in the fetal skin) that may result in intrauterine death. Premature closure of the arterial duct leads to a sudden increase in right ventricular afterload that is not well tolerated by the immature ventricle and may also result in increased systemic venous pressures, high velocity tricuspid and pulmonary regurgitation, fetal hydrops, and death.

\section{HUMAN MODELS OF VENTRICULAR LOADING}

The communications intrinsic to the fetal circulation make it difficult to observe a pure model of pressure or volume loading in the human fetus; obstruction-for example, of the left ventricular outflow-results in both pressure loading of the left ventricle and volume loading of the right because of left to right shunting at the atrial level. Animal models of semi-lunar valve ligation are not representative of human pathophysiology where rate of pressure increase is likely to be less, thus permitting adaptation of the fetal heart to changes in pressure and volume loading. ${ }^{5}$ Adaptive responses in the human fetus have been observed non-invasively using ultrasound Doppler techniques to examine fetal flow patterns and arterial wall pulsations; newer modalities, such as tissue Doppler imaging and assessment of long axis function, may increase our knowledge of the fetal response in the future. ${ }^{6}$

Naturally occurring models of ventricular volume loading usually cause right ventricular volume loading. Common examples include abnormal development of the systemic venous system, such as an absent venous duct or persistent right umbilical vein to the right atrium, and arteriovenous malformations such as vein of Galen aneurysm. Twin-twin transfusion syndrome (TTTS), where monochorionic twins 
share a circulation via placental vascular anastomoses, is probably the best studied model of volume and pressure loading in the human fetus, ${ }^{8-10}$ while semilunar valve stenosis or atresia provides the closest model of ventricular pressure loading in the human fetus.

\section{MYOCARDIAL COMPENSATION AND PROGRESSION TO HEART FAILURE}

Models of systemic pressure loading in the fetal lamb have reported an increase in heart size and weight, predominantly of the right ventricle, with an increase both in number and size of myocardial cells. The increased number of binucleate cells seen suggest a shift to more premature forms that are unable to divide further. ${ }^{11}$ Pathological studies of the recipient twin heart in TTTS are in accord with these animal studies showing increased heart weight and an increase in myocyte number and size. ${ }^{12}$ It appears therefore that the fetus responds to pressure loading by cell division and hypertrophy, but what is the mechanism of decompensation and can it be recognised early before irreversible heart failure ensues? Responses to volume and pressure loading in infancy and childhood include the re-expression and redistribution of substances seen usually only during cardiac modelling, ${ }^{13}$ and suggest a deregulation of cytoskeletal and stress protein expression under conditions of varied haemodynamic imbalance that may contribute to myocardial failure.

The pressure loaded adult heart shows compensation as evidenced by myocyte hypertrophy (but not division) and increased nuclear DNA and Sc-35 (an RNA splicing factor). However, a decrease in the nucleus/cell ratio eventually leads to cellular exhaustion and death. ${ }^{14}$ Pressure loading is associated with upregulation of the angiotensin converting enzyme (ACE) gene (causing local production of angiotensin II) and tissue growth factor (TGF)- $\beta 1$ in both the fetal and adult heart. Both are potent regulators of fibrosis and have a close correlation with myocyte degeneration and death. The renin-angiotensin system is upregulated in the fetus, when circulating volume is reduced as in the donor fetus in TTTS and is thought to play a role in later vascular responses. ${ }^{15}{ }^{16}$ Fibrosis occurs early in response to pressure loading and is a major determinant of diastolic and systolic dysfunction and may form the substrate for arrhythmia. Both fibrosis and calcification are seen in the fetal ventricular wall exposed to high pressure (often estimated $>100 \mathrm{~mm} \mathrm{Hg}$ from regurgitant jets) when there is semilunar valvar stenosis or atresia. It is likely that angiotensin II plays an important role in fetal ventricular decompensation.

\section{CORONARY PERFUSION}

Coronary perfusion is vital for normal ventricular maturation and in the adult heart the pressure induced increase in ventricular mass and fibrosis reduces endocardial perfusion as evidenced by the oncosis of single cells. In the fetus, $75 \%$ of the resistance to coronary flow is caused by intracavity pressure with the rest due to myocardial contraction and tissue pressure. ${ }^{17}$ Hence, elevation of ventricular end diastolic pressure associated with aortic stenosis is a potent determinant of reduced coronary perfusion and the development of fibrosis.

\section{PULMONARY CIRCULATION}

In the adult model of heart failure, pulmonary pressures are raised because of congestion, but in the human fetus abnormalities of the pulmonary bed occur as the aortic valve becomes progressively more stenotic. Pulsed wave Doppler documents that elevation of left ventricular end diastolic pressure reduces transmitral flow and alters pulmonary venous return to the high pressure left atrium. ${ }^{18}$ There is reversal of flow into the pulmonary venous bed (also working at raised pressure) resulting in an increase in vascular smooth muscle and vessel number and hence raised pulmonary vascular resistance. Conversely where flow is reduced, such as in pulmonary atresia, the pulmonary arterial walls are usually hypoplastic. ${ }^{19}$

\section{ROLE OF OXYGEN}

Closure of the oval foramen results in filling of the left ventricle by relatively deoxygenated blood returning from the fetal lungs (instead of from the venous duct), while the oxygen content in the right ventricle and arterial duct is relatively increased. This reduces the materno-fetal oxygen gradient at the placenta and reduces the fetal extraction of oxygen from the placental circulation. This cycle of events does not usually lead to decompensation of the fetal circulation, but is thought to provide one explanation why the deposition of endocardial fibroelastosis appears confined to the left ventricle. ${ }^{20}$

\section{VENTRICULO-VASCULAR COUPLING}

Changes in ventricular pressure or volume loading require appropriate ventriculo-vascular coupling to maintain stroke volume. A sudden rise in intravascular pressure (as seen in the TTTS model of volume loading in the recipient fetus) results in vascular smooth muscle contraction to increase resistance. This is maintained by an increase in medial thickness and then vascular remodelling occurs within weeks to maintain constant wall stress. ${ }^{21}$ Increased volume flow increases elastin deposition and endothelial shear stress, perhaps altering gene expression. ${ }^{22}$ Thus in the recipient fetus of TTTS this combination of factors may explain the altered arterial distensibility measured in childhood. ${ }^{16}$

The developing fetus may respond to reduced nutrition or elevation of end diastolic pressure by a reduction in the deposition of arterial elastin. ${ }^{23}$ Differences in arterial physiology have been observed non-invasively using ultrasound in fetuses with growth restriction caused by placental dysfunction and hence increased impedance. The reduction in pulse amplitude and alteration of other parameters of the arterial wall waveform suggest a reduction of elastic recoil in the fetal arterial wall when distal impedance is increased. However, the long term implications of this on blood pressure are uncertain as no alteration of pulse wave velocity or aortic pulse wave parameters have been measured in childhood. ${ }^{24}$

\section{THE FUTURE: POSSIBLE STRATEGIES TO ALTER PROGRAMMING}

Adult studies have demonstrated that once a certain threshold of ventricular damage is past, postoperative recovery is incomplete. ${ }^{14}$ Raised ventricular pressure and abnormal haemodynamics lead to progressive damage (fibrosis and calcification) in the developing fetal heart and vascular beds. This should be considered in the timing of procedures intended to relieve pressure loading. Abnormality of pulmonary venous flow is also well documented and undoubtedly contributes to the failure of some individuals to adapt optimally in postnatal life. Fetal treatment may prove a beneficial alternative in selected cases allowing earlier intervention and minimising secondary damage to the fetal myocardium and pulmonary vascular bed. ${ }^{25}$

Correspondence to: Dr Helena M Gardiner, Institute of Reproductive and Developmental Biology, Faculty of Medicine, Imperial College, Queen Charlotte's and Chelsea Hospital, Du Cane Road, London, W12 ONN, UK; helena.gardiner@imperial.ac.uk

\section{REFERENCES}

1 Rasanen J, Wood DC, Weiner S, et al. Role of the pulmonary circulation in the distribution of human fetal cardiac output during the second half of pregnancy. Circulation 1996;94:1068-73. 
2 Edelstone DI, Rudolph AM. Preferential streaming of ductus venosus blood to the brain and heart in fetal lambs. Am J Physiology 1979;237:H724-9.

3 Keller BB, Hu N, Tinney JP. Embryonic ventricular diastolic and systolic pressure-volume relation. Cardiology 1994;4:19-27.

4 Gudmundsson S, Marsal K. Umbilical and uteroplacental blood flow velocity waveforms in pregnancies with fetal growth retardation. Eur J Obstet Gynecol R B 1988;27:187-96.

5 Hawkins J, Van Hare GF, Schmidt KG, et al. Effects of increasing afterload on left ventricular output in fetal lambs. Circulation Research 1989;65:127-34.

6 Hecher K, Campbell S, Doyle P, et al. Assessment of fetal compromise by Doppler ultrasound investigation of the fetal circulation. Arterial, intracardiac, and venous blood flow velocity studies. Circulation 1995;91:129-38.

7 Gardiner HM, Brodszki J, Marsal K. Ventriculovascular physiology of the growth restricted fetus. Ultrasound Obstet Gynecol 2001;18:47-53.

8 Hecher K, Plath $\mathrm{H}$, Bregenzer T, et al. Endoscopic laser surgery versus serial amniocenteses in the treatment of severe twin-twin transfusion syndrome. Am J Obstet Gynecol 1999; 180:717-24.

9 Karatza AA, Wolfenden JL, Taylor MJ, et al. Influence of twin-twin transfusion syndrome on fetal cardiovascular structure and function: prospective casecontrol study of 136 monochorionic twin pregnancies. Heart 2002;88:271-7.

10 Fisk NM, Denbow ML, Taylor M, et al. Scientific basis of TTTS and its relevance to treatment strategies. Fetal Diagn Ther 1998;13:49.

11 Barbera A, Giraud GD, Reller MD, et al. Right ventricular systolic pressure load alters myocyte maturation in fetal sheep. Am J Physiol Regul Integr Comp Physiol 2000;279:R1 157-64

12 Naeye R. Organ abnormalities in a human parabiotic syndrome. Am J Path 1965;46:29-34.

13 Gardiner HM, Meng H-X, Dodd S. Varied haemodynamic stress in congenital heart disease causes an alteration in distribution of intermediate filaments and heat shock proteins. J Pathol Suppl 1994;172:[abstract].

14 Hein S, Arnon E, Kostin S, et al. Progression from compensated hypertrophy to failure in the pressure-overloaded human heart: structural deterioration and compensatory mechanisms. Circulation 2003;107:984-91.
15 Mahieu-Caputo D, Dommergues M, Delezoide AL, et al. Twin to twin transfusion syndrome. Role of the fetal renin-angiotensin system. Am J Path 2000;156:629-36.

16 Gardiner HM, Taylor MJO, Karatza AA, et al. Twin-twin transfusion syndrome: the influence of intrauterine laser photocoagulation on arterial distensibility in childhood. Circulation 2003; 107:1906-11.

17 Berne R, Rubio R. Coronary circulation. In: Berne R, Sperelakis N, Geiger S, eds. Handbook of physiology. Section 2: the cardiovascular system. Bethesda, Maryland: American Physiological Society, 1979.

18 Better DJ, Apfel HD, Zidere V, et al. Pattern of pulmonary venous blood flow in the hypoplastic left heart syndrome in the fetus. Heart 2000;81:646-9.

19 Haworth S, Reid L. Quantitative structural study of pulmonary circulation in the new born with aortic atresia, stenosis or coarctation. Thorax 1977;32:121-8.

20 Sharland GK, Chita SK, Fagg NL, et al. Left ventricular dysfunction in the fetus: relation to aortic valve anomalies and endocardial fibroelastosis. $\mathrm{Br}$ Heart $J$ 1991;66:419-24.

21 Fridez P, Makino S, Miyazaki H, et al. Short-term biomechanical adaptation of the rat carotid to acute hypertension: contribution of smooth muscle. Ann Biomed Eng 2001;29:26-34.

22 Durier S, Fassot C, Laurent S, et al. Physiological genomics of human arteries: quantitative relationship between gene expression and arterial stiffness. Circulation 2003;108:1845-51.

23 Martyn CN, Greenwald SE. Impaired synthesis of elastin in walls of the aorta and large conduit arteries during early development as an initiating event in pathogenesis of systemic hypertension. Lancet 1997;350:953-5.

24 Ley D, Stale H, Marsal K. Aortic vessel wall characteristics and blood pressure in children with intrauterine growth retardation and abnormal foetal aortic blood flow. Acta Paediatr 1997;86:299-305.

25 Tulzer G, Arzt W, Franklin RC, et al. Fetal pulmonary valvuloplasty for critical pulmonary stenosis or atresia with intact septum. Lancet 2002;360:1567-8.

\section{IMAGES IN CARDIOLOGY}

\section{Magnetic resonance imaging diagnosing a left ventricular lipoma in a patient with $T$ wave changes on ECG}

A

75 year old woman, with no cardiovascular history, was incidentally found with negative $T$ waves in inferior leads. Echocardiography suspected a nonobstructive hypertrophic cardiomyopathy localised at the inferior wall (measured at $32 \mathrm{~mm}$ ).

Cardiac magnetic resonance imaging (MRI) was performed to confirm the diagnosis and to detect associated ischaemic myocardial disease (1.5T, Signa, General Electric Medical Systems, Milwaukee, USA). Black blood Tl weighted fast spin echo images revealed a well delineated and non-infiltrative high signal intramyocardial ovoid mass of the left ventricle (LV) inferior wall (panel A). Fat suppressed Tl weighted images showed the mass in signal void (panel B). The first pass perfusion with gadolinium and the delayed enhancement sequence showed no perfusion of the cardiac mass. No ischaemic involvement of the myocardium was demonstrated by MRI. These MRI findings established the diagnosis of cardiac lipoma of the inferior LV wall inducing $\mathrm{T}$ wave changes in inferior leads.

Cardiac lipoma is the second most frequent benign tumour of the heart. One quarter of lipomas arise within the myocardium.
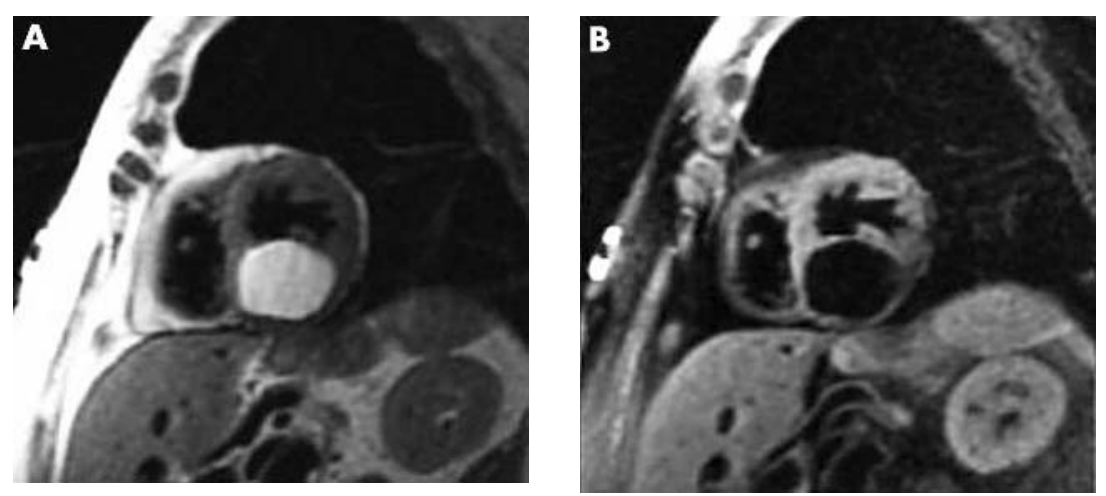

$\mathrm{T} 1$ weighted double inversion recovery fast spin echo image of the heart, short axis view. Image reveals a well delineated, non-

infiltrative, high signal ovoid mass localised within the myocardium of the left ventricular (LV) inferior wall. The LV myocardium is seen in lower signal without hypertrophy.
Fat suppressed image of the same slice shows the ovoid mass of the LV inferior wall in signal void, suggesting the diagnosis of cardiac lipoma.
A Azarine

S Castela

E Mousseaux

aazarine@free.fr 